\title{
On Hermite-Hadamard-Fejér type inequalities for convex functions via fractional integrals
}

\author{
AbDullah AKKuRT AND HÜSEYIN Yildirim
}

\begin{abstract}
In this paper, we have established some generalized integral inequalities of Hermite-Hadamard-Fejér type for generalized fractional integrals. The results presented here would provide generalizations of those given in earlier works.
\end{abstract}

\section{INTRODUCTION}

Let $f: I \subset \mathbb{R} \rightarrow \mathbb{R}$ be a convex function define on an interval $I$ of real numbers, and $a, b \in I$ with $a<b$. Then the following inequalities hold:

$$
f\left(\frac{a+b}{2}\right) \leq \frac{1}{b-a} \int_{a}^{b} f(x) \mathrm{d} x \leq \frac{f(a)+f(b)}{2} .
$$

It was first discovered by Hermite in 1881 in the Journal Mathesis. This inequality (1) was nowhere mentioned in the mathematical literature untill 1893. In [4], Beckenbach, a leading expert on the theory of convex functions, wrote that the inequality (1) was proved by Hadamard in 1893. In 1974, Mitrinovič found Hermite and Hadamard's note in Mathesis. That is why, the inequality (1) was known as Hermite-Hadamard inequality. We note that Hermite-Hadamard's inequality may be regarded as a refinements of the concept of convexity and it follows easily from Jensen's inequality. This inequality (1) has been received renewed attention in recent years and a remarkable variety of refinements and generalizations have been found in [4]-[16], [18], [20].

The most well known inequalities connected with the integral mean of a convex functions are Hermite-Hadamard inequalities or its weighted versions, the so-called Hermite-Hadamard-Fejěr inequality.

In [10], Fejér established the following Fejér inequality which is the weighted generalization of Hermite-Hadamard inequalities (1):

2010 Mathematics Subject Classification. Primary: 26A33, 26A51; Secondary: 26D15.

Key words and phrases. Integral inequalities, Fractional integrals, Hermite-HadamardFejér Inequality. 
Theorem 1.1. Let $f: I \rightarrow \mathbb{R}$ be a convex on $I$ and let $a, b \in I$ with $a<b$. Then the inequality

$$
f\left(\frac{a+b}{2}\right) \int_{a}^{b} g(x) \mathrm{d} x \leq \frac{1}{b-a} \int_{a}^{b} f(t) g(x) \mathrm{d} t \leq \frac{f(a)+f(b)}{2} \int_{a}^{b} g(x) \mathrm{d} x .
$$

holds, where $f:[a, b] \rightarrow \mathbb{R}$ is nonnegative, integrable, and symmetric to $\frac{a+b}{2}$.

In [11], Sarikaya et al. represented Hermite-Hadamard's inequalities in fractional integral forms as follows.

Theorem 1.2. Let $f:[a, b] \rightarrow \mathbb{R}$ be a positive function with $0 \leq a<b$ and $f \in L[a, b]$. If $f$ is a convex function on $[a, b]$, then the following inequalities for fractional integrals holds

$$
f\left(\frac{a+b}{2}\right) \leq \frac{\Gamma(\alpha+1)}{2(b-a)^{\alpha}}\left[J_{a^{+}}^{\alpha} f(b)+J_{b^{-}}^{\alpha} f(a)\right] \leq \frac{f(a)+f(b)}{2},
$$

with $\alpha>0$.

In [5] Set et. al. obtained the following lemma.

Lemma 1.1. Let $f:[a, b] \rightarrow \mathbb{R}$ be a differentiable mapping on $(a, b)$ with $a<b$ and let $g:[a, b] \rightarrow \mathbb{R}$. If $f^{\prime}, g \in L[a, b]$, then the following identity for fractional integrals holds:

$$
\begin{aligned}
f\left(\frac{a+b}{2}\right) & {\left[J_{\left(\frac{a+b}{2}\right)^{-}}^{\alpha} g(a)+J_{\left(\frac{a+b}{2}\right)^{+}}^{\alpha} g(b)\right] } \\
- & {\left[J_{\left(\frac{a+b}{2}\right)^{-}}^{\alpha}(g f)(a)+J_{\left(\frac{a+b}{2}\right)^{+}}^{\alpha}(f g)(b)\right]=\frac{1}{\Gamma(\alpha)} \int_{a}^{b} k(t) \mathrm{d} f(t), }
\end{aligned}
$$

where

$$
k(t)= \begin{cases}\int_{a}^{t}(s-a)^{\alpha-1} g(s) \mathrm{d} s, & t \in\left[a, \frac{a+b}{2}\right], \\ \int_{b}^{t}(b-s)^{\alpha-1} g(s) \mathrm{d} s, & t \in\left[\frac{a+b}{2}, b\right] .\end{cases}
$$

We give some necessary definitions and mathematical preliminaries of fractional calculus theory which are used throughout this paper.

Definition 1.1. Let $h(x)$ be an increasing and positive monotone function on $[0, \infty)$, also derivative $h^{\prime}(x)$ is continuous on $[0, \infty)$ and $h(0)=0$. 
The space $X_{h}^{p}(0, \infty)(1 \leq p<\infty)$ of those real-valued Lebesque measurable functions $f$ on $[0, \infty)$ for which

$$
\|f\|_{X_{h}^{p}}=\left(\int_{0}^{\infty}|f(t)|^{p} h^{\prime}(x) \mathrm{d} t\right)^{\frac{1}{p}}<\infty, \quad 1 \leq p \leq \infty
$$

and for the case $p=\infty$

$$
\|f\|_{X_{h}^{\infty}}=\operatorname{ess} \sup _{1 \leq t<\infty}\left[f(t) h^{\prime}(x)\right] .
$$

Definition 1.2. ([6]). In particular, when $h(x)=x(1 \leq p<\infty)$ the space $X_{h}^{p}(0, \infty)$ coincides with the $L_{p}[0, \infty)$-space $\left(\|f\|_{X_{h}^{\infty}}=\|f\|_{\infty}\right)$ and also if we take $h(x)=\frac{x^{k+1}}{k+1}(k \geq 0)$ the space $X_{h}^{p}(0, \infty)$ coincides with the $L_{p, k}[0, \infty)$-space.

Definition 1.3. ([1]). Let $(a, b)$ be a finite interval of the real line $\mathbb{R}$ and $\alpha>0$. Also let $h(x)$ be an increasing and positive monotone function on $(a, b]$, having a continuous derivative $h^{\prime}(x)$ on $(a, b)$. The left- and rightsided fractional integrals of a function $f$ with respect to another function $h$ on $[a, b]$ are defined by

$$
\left(J_{a^{+}, h}^{\alpha} f\right)(x):=\frac{1}{\Gamma(\alpha)} \int_{a}^{x}[h(x)-h(t)]^{\alpha-1} h^{\prime}(t) f(t) \mathrm{d} t, \quad x \geq a
$$

and

$$
\left(J_{b^{-}, h}^{\alpha} f\right)(x):=\frac{1}{\Gamma(\alpha)} \int_{x}^{b}[h(t)-h(x)]^{\alpha-1} h^{\prime}(t) f(t) \mathrm{d} t, \quad x \leq b .
$$

Definition 1.4. If we take $h(x)=x$, then the equalities (7) and (8) will be

$$
\left(J_{a^{+}}^{\alpha} f\right)(x)=\frac{1}{\Gamma(\alpha)} \int_{a}^{x}(x-t)^{\alpha-1} f(t) \mathrm{d} t, \quad x>a
$$

and

$$
\left(J_{b^{-}}^{\alpha} f\right)(x)=\frac{1}{\Gamma(\alpha)} \int_{x}^{b}(t-x)^{\alpha-1} f(t) \mathrm{d} t, \quad b>x .
$$

These integrals are called left-sided Riemann-Liouville fractional integral and right-sided Riemann-Liouville fractional integral respectively [1]-[3], [6], [17].

In this paper, we have established some generalized fractional integral inequalities. The results presented here would provide generalizations of those given in earlier works. 


\section{Main Results}

Lemma 2.1. Let $f:[a, b] \rightarrow \mathbb{R}$ be a differentiable mapping on $(a, b)$ with $a<b$ and let $g:[a, b] \rightarrow \mathbb{R}$. If $f^{\prime}, g \in X_{h}^{p}[a, b]$, then the following identity for fractional integrals holds:

$$
\begin{aligned}
& f\left(h\left(\frac{a+b}{2}\right)\right)\left[J_{\left(\frac{a+b}{2}\right)^{-}}^{\alpha} g(h(a))+J_{\left(\frac{a+b}{2}\right)^{+}}^{\alpha} g(h(b))\right] \\
& -\left[J_{\left(\frac{a+b}{2}\right)^{-}}^{\alpha}(g \times(f \circ h))(a)+J_{\left(\frac{a+b}{2}\right)^{+}}^{\alpha}(g \times(f \circ h))(b)\right] \\
& =\frac{1}{\Gamma(\alpha)} \int_{a}^{b} k(t) \mathrm{d} f(h(t))
\end{aligned}
$$

where

$$
k(t)= \begin{cases}\int_{a}^{t}(h(s)-h(a))^{\alpha-1} g(s) h^{\prime}(s) \mathrm{d} s, & t \in\left[a, \frac{a+b}{2}\right], \\ \int_{b}^{t}(h(b)-h(s))^{\alpha-1} g(s) h^{\prime}(s) \mathrm{d} s, & t \in\left[\frac{a+b}{2}, b\right] .\end{cases}
$$

Proof. It suffices to note that

$$
\begin{aligned}
I= & \int_{a}^{b} k(t) \mathrm{d} f(h(t)) \\
= & \int_{a}^{\frac{a+b}{2}}\left(\int_{a}^{t}(h(s)-h(a))^{\alpha-1} g(s) h^{\prime}(s) \mathrm{d} s\right) \mathrm{d} f(h(t)) \\
& +\int_{\frac{a+b}{2}}^{b}\left(\int_{b}^{t}(h(b)-h(s))^{\alpha-1} g(s) h^{\prime}(s) \mathrm{d} s\right) \mathrm{d} f(h(t)) \\
= & I_{1}+I_{2} .
\end{aligned}
$$

By integration by parts, we get

$$
\begin{aligned}
I_{1} & =\int_{a}^{\frac{a+b}{2}}\left(\int_{a}^{t}(h(s)-h(a))^{\alpha-1} g(s) h^{\prime}(s) \mathrm{d} s\right) \mathrm{d} f(h(t)) \\
& =\left.\left(\int_{a}^{t}(h(s)-h(a))^{\alpha-1} g(s) h^{\prime}(s) \mathrm{d} s\right) f(h(t))\right|_{a} ^{\frac{a+b}{2}}
\end{aligned}
$$




$$
\begin{aligned}
& -\int_{a}^{\frac{a+b}{2}}(h(t)-h(a))^{\alpha-1} g(t) f(h(t)) h^{\prime}(t) \mathrm{d} t \\
= & f\left(h\left(\frac{a+b}{2}\right)\right) \int_{a}^{\frac{a+b}{2}}(h(s)-h(a))^{\alpha-1} g(s) h^{\prime}(s) \mathrm{d} s \\
& -\int_{a}^{\frac{a+b}{2}}(h(t)-h(a))^{\alpha-1} g(t) f(h(t)) h^{\prime}(t) \mathrm{d} t \\
= & \Gamma(\alpha)\left[f\left(h\left(\frac{a+b}{2}\right)\right) J_{\left(\frac{a+b}{2}\right)^{-}}^{\alpha} g(h(a))-J_{\left(\frac{a+b}{2}\right)^{-}}^{\alpha}(g(f \circ h))(a)\right]
\end{aligned}
$$

and similarly

$$
\begin{aligned}
& I_{2}=\int_{\frac{a+b}{2}}^{b}\left(\int_{b}^{t}(h(b)-h(s))^{\alpha-1} g(s) h^{\prime}(s) \mathrm{d} s\right) \mathrm{d} f(h(t)) \\
& =\left.\left(\int_{b}^{t}(h(s)-h(a))^{\alpha-1} g(s) h^{\prime}(s) \mathrm{d} s\right) f(h(t))\right|_{\frac{a+b}{2}} ^{b} \\
& -\int_{\frac{a+b}{2}}^{b}(h(b)-h(t))^{\alpha-1} g(t) f(h(t)) h^{\prime}(t) \mathrm{d} t \\
& =f\left(h\left(\frac{a+b}{2}\right)\right) \int_{\frac{a+b}{2}}^{b}(h(b)-h(s))^{\alpha-1} g(s) h^{\prime}(s) \mathrm{d} s \\
& -\int_{\frac{a+b}{2}}^{b}(h(b)-h(t))^{\alpha-1} g(t) f(h(t)) h^{\prime}(t) \mathrm{d} t \\
& =\Gamma(\alpha)\left[f\left(h\left(\frac{a+b}{2}\right)\right) J_{\left(\frac{a+b}{2}\right)^{+}}^{\alpha} g(h(b))-J_{\left(\frac{a+b}{2}\right)^{+}}^{\alpha}(g(f \circ h))(b)\right] .
\end{aligned}
$$

Thus, can write

$$
\begin{aligned}
I= & I_{1}+I_{2} \\
= & \Gamma(\alpha)\left\{f\left(h\left(\frac{a+b}{2}\right)\right)\left[J_{\left(\frac{a+b}{2}\right)^{-}}^{\alpha} g(h(a))+J_{\left(\frac{a+b}{2}\right)^{+}}^{\alpha} g(h(b))\right]\right. \\
& \left.-\left[J_{\left(\frac{a+b}{2}\right)^{-}}^{\alpha}(g(f \circ h))(a)+J_{\left(\frac{a+b}{2}\right)^{\alpha}}^{\alpha}(g(f \circ h))(b)\right]\right\} .
\end{aligned}
$$


Multiplying the both sides $(\Gamma(\alpha))^{-1}$, we obtain (11) which complates the proof.

Remark 2.1. If we choose $h(x)=x$ in Lemma 2.1, then the inequality (11) reduces to (1.4) .

Remark 2.2. If we choose $h(x)=x, g(x)=1$ and $\alpha=1$ in Lemma 2.1, we obtain Lemma 2.1 in [21].

Theorem 2.1. Let $f: I \rightarrow \mathbb{R}$ be a differentiable mapping on $I^{\circ}$ and $f^{\prime} \in$ $X_{h}^{p}[a, b]$ with $a<b$ and $g:[a, b] \rightarrow \mathbb{R}$ is continuous. If $\left|f^{\prime}\right|$ is convex on $[a, b]$, then the following inequality for fractional integrals holds:

$$
\begin{aligned}
& \mid f\left(h\left(\frac{a+b}{2}\right)\right)\left[J_{\left(\frac{a+b}{2}\right)^{-}}^{\alpha} g(h(a))+J_{\left(\frac{a+b}{2}\right)^{+}}^{\alpha} g(h(b))\right] \\
& -\left[J_{\left(\frac{a+b}{2}\right)^{-}}^{\alpha}(g \times(f \circ h))(a)+J_{\left(\frac{a+b}{2}\right)^{+}}^{\alpha}(g \times(f \circ h))(b)\right] \mid \\
& \leq \frac{|| g \|_{X_{h}^{\infty}\left[a, \frac{a+b}{2}\right], \infty}}{(h(b)-h(a)) \Gamma(\alpha+1)}\left\{| f ^ { \prime } ( h ( a ) ) | \left[\frac{\left(h\left(\frac{a+b}{2}\right)-h(a)\right)^{\alpha+1}}{\alpha+1}(h(b)-h(a))\right.\right. \\
& \left.\quad-\left|f^{\prime}(h(a))\right| \frac{\left(h\left(\frac{a+b}{2}\right)-h(a)\right)^{\alpha+2}}{\alpha+2}\right] \\
& \left.+\left|f^{\prime}(h(b))\right|\left[\frac{\left(h\left(\frac{a+b}{2}\right)-h(a)\right)^{\alpha+2}}{\alpha+2}\right]\right\}+\frac{\|g\|_{X_{h}^{\infty}\left[\frac{a+b}{2}, b\right], \infty}}{(h(b)-h(a)) \Gamma(\alpha+1)} \\
& \times\left\{\left|f^{\prime}(h(b))\right|\left[\frac{\left(h(b)-h\left(\frac{a+b}{2}\right)\right)^{\alpha+1}}{\alpha+1}(h(b)-h(a))-\frac{\left(h(b)-h\left(\frac{a+b}{2}\right)\right)^{\alpha+2}}{\alpha+2}\right]\right. \\
& \left.\quad+\left|f^{\prime}(h(a))\right|\left[\frac{\left(h(b)-h\left(\frac{a+b}{2}\right)\right)^{\alpha+2}}{\alpha+2}\right]\right\}
\end{aligned}
$$

with $\alpha>0$.

Proof. If $\left|f^{\prime}\right|$ is convex on $[a, b]$, we know that for $t \in[a, b]$

$$
\begin{aligned}
& \left|f^{\prime}(h(t))\right| \\
= & \left|f^{\prime}\left(\frac{h(b)-h(t)}{h(b)-h(a)} h(a)+\frac{h(t)-h(a)}{h(b)-h(a)} h(b)\right)\right| \\
\leq & \frac{h(b)-h(t)}{h(b)-h(a)}\left|f^{\prime}(h(a))\right|+\frac{h(t)-h(a)}{h(b)-h(a)}\left|f^{\prime}(h(b))\right| .
\end{aligned}
$$

From Lemma 2.1, we have

$$
\mid f\left(h\left(\frac{a+b}{2}\right)\right)\left[J_{\left(\frac{a+b}{2}\right)^{\alpha}}^{\alpha} g(h(a))+J_{\left(\frac{a+b}{2}\right)^{+}}^{\alpha} g(h(b))\right]
$$




$$
\begin{aligned}
& -\left[J_{\left(\frac{a+b}{2}\right)^{\alpha}}^{\alpha}(g(f \circ h))(a)+J_{\left(\frac{a+b}{2}\right)^{+}}^{\alpha}(g(f \circ h))(b)\right] \mid \\
& \leq \frac{1}{\Gamma(\alpha)}\left\{\int_{a}^{\frac{a+b}{2}}\left|\int_{a}^{t}(h(s)-h(a))^{\alpha-1} g(s) h^{\prime}(s) \mathrm{d} s\right|\left|f^{\prime}(h(t))\right| h^{\prime}(t) \mathrm{d} t\right. \\
& \left.+\int_{\frac{a+b}{2}}^{b}\left|\int_{b}^{t}(h(b)-h(s))^{\alpha-1} g(s) h^{\prime}(s) \mathrm{d} s\right|\left|f^{\prime}(h(t))\right| h^{\prime}(t) \mathrm{d} t\right\} \\
& \leq \frac{\|g\|_{X_{h}^{\infty}\left[a, \frac{a+b}{2}\right], \infty}}{(h(b)-h(a)) \Gamma(\alpha)}\left\{\int_{a}^{\frac{a+b}{2}}\left|\int_{a}^{t}(h(s)-h(a))^{\alpha-1} h^{\prime}(s) \mathrm{d} s\right|\right. \\
& \times\left(h(b)-h(t)\left|f^{\prime}(h(a))\right|\right) h^{\prime}(t) \mathrm{d} t \\
& \left.+\int_{a}^{\frac{a+b}{2}}\left|\int_{a}^{t}(h(s)-h(a))^{\alpha-1} h^{\prime}(s) d s\right|\left(h(t)-h(a)\left|f^{\prime}(h(b))\right|\right) h^{\prime}(t) \mathrm{d} t\right\} \\
& +\frac{\|g\|_{X_{h}^{\infty}\left[\frac{a+b}{2}, b\right], \infty}}{(h(b)-h(a)) \Gamma(\alpha)}\left\{\int_{\frac{a+b}{2}}^{b}\left|\int_{b}^{t}(h(b)-h(s))^{\alpha-1} h^{\prime}(s) d s\right|\right. \\
& \times\left(h(b)-h(t)\left|f^{\prime}(h(a))\right|\right) h^{\prime}(t) \mathrm{d} t \\
& \left.+\int_{\frac{a+b}{2}}^{b}\left|\int_{b}^{t}(h(b)-h(s))^{\alpha-1} h^{\prime}(s) \mathrm{d} s\right|\left(h(t)-h(a)\left|f^{\prime}(h(b))\right|\right) h^{\prime}(t) \mathrm{d} t\right\} . \\
& \leq \frac{\|g\|_{X_{h}^{\infty}\left[a, \frac{a+b}{2}\right], \infty}}{(h(b)-h(a)) \Gamma(\alpha+1)}\left\{\int_{a}^{\frac{a+b}{2}}(h(t)-h(a))^{\alpha}\left(h(b)-h(t)\left|f^{\prime}(h(a))\right|\right) h^{\prime}(t) \mathrm{d} t\right. \\
& \left.+\int_{a}^{\frac{a+b}{2}}(h(t)-h(a))^{\alpha+1}\left|f^{\prime}(h(b))\right| h^{\prime}(t) \mathrm{d} t\right\} \\
& +\frac{\|g\|_{X_{h}^{\infty}\left[\frac{a+b}{2}, b\right], \infty}}{(h(b)-h(a)) \Gamma(\alpha+1)}\left\{\int_{\frac{a+b}{2}}^{b}(h(b)-h(t))^{\alpha+1}\left|f^{\prime}(h(a))\right| h^{\prime}(t) \mathrm{d} t\right.
\end{aligned}
$$




$$
\begin{aligned}
& \left.+\int_{\left.\frac{a+b}{2}(h(b)-h(t))^{\alpha-1}\left(h(t)-h(a)\left|f^{\prime}(h(b))\right|\right) h^{\prime}(t) \mathrm{d} t\right\}}^{b}\right\} \\
& \leq \frac{\|g\|_{X_{h}^{\infty}\left[a, \frac{a+b}{2}\right], \infty}}{(h(b)-h(a)) \Gamma(\alpha+1)}\left\{| f ^ { \prime } ( h ( a ) ) | \left[\frac{\left(h\left(\frac{a+b}{2}\right)-h(a)\right)^{\alpha+1}}{\alpha+1}(h(b)-h(a))\right.\right. \\
& \left.\left.-\frac{\left(h\left(\frac{a+b}{2}\right)-h(a)\right)^{\alpha+2}}{\alpha+2}\right]+\left|f^{\prime}(h(b))\right|\left[\frac{\left(h\left(\frac{a+b}{2}\right)-h(a)\right)^{\alpha+2}}{\alpha+2}\right]\right\} \\
& +\frac{\|g\|_{X_{h}^{\infty}\left[\frac{a+b}{2}, b\right], \infty}}{(h(b)-h(a)) \Gamma(\alpha+1)}\left\{\left|f^{\prime}(h(b))\right|\left[\frac{\left(h(b)-h\left(\frac{a+b}{2}\right)\right)^{\alpha+1}}{\alpha+1}(h(b)-h(a))\right]\right. \\
& \left.-\frac{\left(h(b)-h\left(\frac{a+b}{2}\right)\right)^{\alpha+2}}{\alpha+2}+\left|f^{\prime}(h(a))\right|\left[\frac{\left(h(b)-h\left(\frac{a+b}{2}\right)\right)^{\alpha+2}}{\alpha+2}\right]\right\} .
\end{aligned}
$$

This completes the proof.

Remark 2.3. If we choose $h(x)=x$ in Theorem 2.1, we obtain Theorem 6 in [5].

Remark 2.4. If we choose $h(x)=x, g(x)=1$ and $\alpha=1$ in Theorem 2.1, we obtain Theorem 2.2 in [21].

Theorem 2.2. Let $f: I \rightarrow \mathbb{R}$ be a differentiable mapping on $I^{\circ}$ and $f^{\prime} \in$ $X_{h}^{p}[a, b]$ with $a<b$ and $g:[a, b] \rightarrow \mathbb{R}$ is continuous. If $\left|f^{\prime}\right|^{q}$ is convex on $[a, b], q \geq 1$ then the following inequality for fractional integrals holds:

$$
\begin{aligned}
& f\left(h\left(\frac{a+b}{2}\right)\right)\left[J_{\left(\frac{a+b}{2}\right)^{-}}^{\alpha} g(h(a))+J_{\left(\frac{a+b}{2}\right)^{+}}^{\alpha} g(h(b))\right] \\
& -\left[J_{\left(\frac{a+b}{2}\right)^{-}}^{\alpha}(g \times(f \circ h))(a)+J_{\left(\frac{a+b}{2}\right)^{+}}^{\alpha}(g \times(f \circ h))(b)\right] \\
& \leq \frac{\|g\|_{X_{h}^{\infty}\left[a, \frac{a+b}{2}\right], \infty}}{[(h(b)-h(a))]^{\frac{1}{q}} \Gamma(\alpha)}\left(\frac{h\left(\frac{a+b}{2}\right)-h(a)}{\alpha(\alpha+1)}\right)^{1-\frac{1}{q}}\left\{\left|f^{\prime}(h(a))\right|^{q}\right. \\
& \times\left[\frac{\left(h\left(\frac{a+b}{2}\right)-h(a)\right)^{\alpha+1}(h(b)-h(a))}{\alpha+1}-\frac{\left(h\left(\frac{a+b}{2}\right)-h(a)\right)^{\alpha+2}}{\alpha+2}\right] \\
& \left.+\frac{\left|f^{\prime}(h(b))\right|^{q}\left(h\left(\frac{a+b}{2}\right)-h(a)\right)^{\alpha+2}}{\alpha+2}\right\}^{\frac{1}{q}} \\
& +\frac{\|g\|_{X_{h}^{\infty}\left[\frac{a+b}{2}, b\right], \infty}}{[(h(b)-h(a))]^{\frac{1}{q}} \Gamma(\alpha)}\left(\frac{h(b)-h\left(\frac{a+b}{2}\right)}{\alpha(\alpha+1)}\right)^{1-\frac{1}{q}}\left\{\left|f^{\prime}(h(b))\right|^{q}\right.
\end{aligned}
$$




$$
\begin{aligned}
& \times\left[\frac{\left(h(b)-h\left(\frac{a+b}{2}\right)\right)^{\alpha+1}(h(b)-h(a))}{\alpha+1}-\frac{\left(h(b)-h\left(\frac{a+b}{2}\right)\right)^{\alpha+2}}{\alpha+2}\right] \\
& \left.+\left|f^{\prime}(h(a))\right|^{q}\left[\frac{\left(h(b)-h\left(\frac{a+b}{2}\right)\right)^{\alpha+2}}{\alpha+2}\right]\right\}^{\frac{1}{q}} .
\end{aligned}
$$

with $\alpha>0$.

Proof. If $\left|f^{\prime}\right|^{q}$ is convex on $[a, b]$, we know that for $t \in[a, b]$

$$
\begin{aligned}
\left|f^{\prime}(h(t))\right|^{q} & =\left|f^{\prime}\left(\frac{h(b)-h(t)}{h(b)-h(a)} h(a)+\frac{h(t)-h(a)}{h(b)-h(a)} h(b)\right)\right|^{q} \\
& \leq \frac{h(b)-h(t)}{h(b)-h(a)}\left|f^{\prime}(h(a))\right|^{q}+\frac{h(t)-h(a)}{h(b)-h(a)}\left|f^{\prime}(h(b))\right|^{q} .
\end{aligned}
$$

From Lemma 2.1, power mean inequality and the convexity of $\left|f^{\prime}\right|^{q}$, it follows that

$$
\begin{aligned}
& f\left(h\left(\frac{a+b}{2}\right)\right)\left[J_{\left(\frac{a+b}{2}\right)^{-}}^{\alpha} g(h(a))+J_{\left(\frac{a+b}{2}\right)^{+}}^{\alpha} g(h(b))\right] \\
& -\left[J_{\left(\frac{a+b}{2}\right)^{-}}^{\alpha}(g(f \circ h))(a)+J_{\left(\frac{a+b}{2}\right)^{+}}^{\alpha}(g(f \circ h))(b)\right] \\
& \leq \frac{1}{\Gamma(\alpha)}\left\{\left(\int_{a}^{\frac{a+b}{2}}\left|\int_{a}^{t}(h(s)-h(a))^{\alpha-1} g(s) h^{\prime}(s) \mathrm{d} s\right| h^{\prime}(t) \mathrm{d} t\right)^{1-1 / q}\right. \\
& \left.\times\left(\int_{a}^{\frac{a+b}{2}}\left|\int_{a}^{t}(h(s)-h(a))^{\alpha-1} g(s) h^{\prime}(s) \mathrm{d} s\right|\left|f^{\prime}(h(t))\right|^{q} h^{\prime}(t) \mathrm{d} t\right)^{1 / q}\right\} \\
& +\frac{1}{\Gamma(\alpha)}\left\{\left(\int_{\frac{a+b}{2}}^{b}\left|\int_{b}^{t}(h(b)-h(s))^{\alpha-1} g(s) h^{\prime}(s) \mathrm{d} s\right| h^{\prime}(t) \mathrm{d} t\right)^{1-1 / q}\right. \\
& \left.\times\left(\int_{\frac{a+b}{2}}^{b}\left|\int_{b}^{t}(h(b)-h(s))^{\alpha-1} g(s) h^{\prime}(s) \mathrm{d} s\right|\left|f^{\prime}(h(t))\right|^{q} h^{\prime}(t) \mathrm{d} t\right)^{1 / q}\right\} \\
& \leq \frac{\|g\|_{X_{h}^{\infty}\left[a, \frac{a+b}{2}\right], \infty}}{\Gamma(\alpha)}\left\{\left(\int_{a}^{\frac{a+b}{2}}\left|\int_{a}^{t}(h(s)-h(a))^{\alpha-1} h^{\prime}(s) \mathrm{d} s\right| h^{\prime}(t) \mathrm{d} t\right)^{1-1 / q}\right.
\end{aligned}
$$




$$
\begin{aligned}
& \left.\times\left(\int_{a}^{\frac{a+b}{2}}\left|\int_{a}^{t}(h(s)-h(a))^{\alpha-1} h^{\prime}(s) \mathrm{d} s\right|\left|f^{\prime}(h(t))\right|^{q} h^{\prime}(t) \mathrm{d} t\right)^{1 / q}\right\} \\
& +\frac{\|g\|_{X_{h}^{\infty}\left[\frac{a+b}{2}, b\right], \infty}}{\Gamma(\alpha)}\left\{\left(\int_{\frac{a+b}{2}}\left|\int_{b}^{t}(h(b)-h(s))^{\alpha-1} h^{\prime}(s) \mathrm{d} s\right| h^{\prime}(t) \mathrm{d} t\right)^{1-1 / q}\right. \\
& \left.\times\left(\int_{\frac{a+b}{2}}^{b}\left|\int_{b}^{t}(h(b)-h(s))^{\alpha-1} h^{\prime}(s) \mathrm{d} s\right|\left|f^{\prime}(h(t))\right|^{q} h^{\prime}(t) \mathrm{d} t\right)^{1 / q}\right\} . \\
& \leq \frac{\|g\|_{X_{h}^{\infty}\left[a, \frac{a+b}{2}\right], \infty}}{[(h(b)-h(a))]^{\frac{1}{q}} \Gamma(\alpha)}\left\{\int_{a}^{\frac{a+b}{2}}\left|\int_{a}^{t}(h(s)-h(a))^{\alpha-1} h^{\prime}(s) \mathrm{d} s\right|\right. \\
& \times\left(h(b)-h(t)\left|f^{\prime}(h(a))\right|^{q}\right) h^{\prime}(t) \mathrm{d} t \\
& \left.+\int_{a}^{\frac{a+b}{2}}\left|\int_{a}^{t}(h(s)-h(a))^{\alpha-1} h^{\prime}(s) d s\right|\left(h(t)-h(a)\left|f^{\prime}(h(b))\right|^{q}\right) h^{\prime}(t) \mathrm{d} t\right\}^{\frac{1}{q}} \\
& +\frac{\|g\|_{X_{h}^{\infty}\left[\frac{a+b}{2}, b\right], \infty}}{[(h(b)-h(a))]^{\frac{1}{q}} \Gamma(\alpha)}\left\{\int_{\frac{a+b}{2}}^{b}\left|\int_{b}^{t}(h(b)-h(s))^{\alpha-1} h^{\prime}(s) \mathrm{d} s\right|\right. \\
& \times\left(h(b)-h(t)\left|f^{\prime}(h(a))\right|^{q}\right) h^{\prime}(t) \mathrm{d} t \\
& \left.+\int_{\frac{a+b}{2}}^{b}\left|\int_{b}^{t}(h(b)-h(s))^{\alpha-1} h^{\prime}(s) \mathrm{d} s\right|\left(h(t)-h(a)\left|f^{\prime}(h(b))\right|^{q}\right) h^{\prime}(t) \mathrm{d} t\right\}^{\frac{1}{q}} \\
& \leq \frac{\|g\|_{X_{h}^{\infty}\left[a, \frac{a+b}{2}\right], \infty}}{[(h(b)-h(a))]^{\frac{1}{q}} \Gamma(\alpha)}\left(\frac{h\left(\frac{a+b}{2}\right)-h(a)}{\alpha(\alpha+1)}\right)^{1-1 / q} \\
& \times\left\{\left[\frac{\left|f^{\prime}(h(a))\right|^{q}\left(h\left(\frac{a+b}{2}\right)-h(a)\right)^{\alpha+1}(h(b)-h(a))}{\alpha+1}\right.\right. \\
& \left.\left.-\frac{\left|f^{\prime}(h(a))\right|^{q}\left(h\left(\frac{a+b}{2}\right)-h(a)\right)^{\alpha+2}}{\alpha+2}\right]+\left|f^{\prime}(h(b))\right|^{q}\left[\frac{\left(h\left(\frac{a+b}{2}\right)-h(a)\right)^{\alpha+2}}{\alpha+2}\right]\right\}^{\frac{1}{q}}
\end{aligned}
$$




$$
\begin{aligned}
& +\frac{\|g\|_{X_{h}^{\infty}\left[\frac{a+b}{2}, b\right], \infty}}{[(h(b)-h(a))]^{\frac{1}{q}} \Gamma(\alpha)}\left(\frac{h(b)-h\left(\frac{a+b}{2}\right)}{\alpha(\alpha+1)}\right)^{1-1 / q} \\
& \times\left\{| f ^ { \prime } ( h ( b ) ) | ^ { q } \left[\frac{\left(h(b)-h\left(\frac{a+b}{2}\right)\right)^{\alpha+1}(h(b)-h(a))}{\alpha+1}\right.\right. \\
& \left.\left.\quad-\frac{\left(h(b)-h\left(\frac{a+b}{2}\right)\right)^{\alpha+2}}{\alpha+2}\right]+\left|f^{\prime}(h(a))\right|^{q}\left[\frac{\left(h(b)-h\left(\frac{a+b}{2}\right)\right)^{\alpha+2}}{\alpha+2}\right]\right\}^{\frac{1}{q}} .
\end{aligned}
$$

Remark 2.5. If we choose $h(x)=x$ in Theorem 2.2, we obtain Theorem 7 in [5].

Lemma 2.2. Let $f:[a, b] \rightarrow \mathbb{R}$ be a differentiable mapping on $(a, b)$ with $a<b$ and let $g:[a, b] \rightarrow \mathbb{R}$. If $f^{\prime}, g \in X_{h}^{p}[a, b]$, then the following identity for fractional integrals holds:

$$
\begin{aligned}
& \left\{f ( h ( \frac { a + b } { 2 } ) ) \left[-J_{a^{+}}^{\alpha} g(h(b))-J_{b^{-}}^{\alpha} g(h(a))\right.\right. \\
& \quad+J_{\left(\frac{a+b}{2}\right)^{+}}^{\alpha} g(h(b))+J_{\left(\frac{a+b}{2}\right)^{-}}^{\alpha} g(h(a)) \\
& \left.\quad+\frac{1}{\Gamma(\alpha)} \int_{a}^{b}(h(b)-h(a))^{\alpha-1} g(s) h^{\prime}(s) \mathrm{d} s\right] \\
& \quad+J_{a^{+}}^{\alpha}(g(f \circ h))(b)+J_{b^{-}}^{\alpha}(g(f \circ h))(a) \\
& \quad-J_{\left(\frac{a+b}{2}\right)^{+}}^{\alpha}(g(f \circ h))(b)-J_{\left(\frac{a+b}{2}\right)^{-}}^{\alpha}(g(f \circ h))(a) \\
& \left.\quad-\frac{1}{\Gamma(\alpha)} \int_{a}^{b}(h(b)-h(a))^{\alpha-1} g(t) f(h(t)) h^{\prime}(t) \mathrm{d} t\right\} \\
& =\frac{1}{\Gamma(\alpha)} \int_{a}^{b} k(t) \mathrm{d} f(h(t))
\end{aligned}
$$

where

$$
k(t)=\left\{\begin{aligned}
\int_{a}^{t}\left[(h(b)-h(a))^{\alpha-1}-(h(b)-h(s))^{\alpha-1}\right. & \\
& \left.+(h(s)-h(a))^{\alpha-1}\right] g(s) h^{\prime}(s) \mathrm{d} s \quad t \in\left[a, \frac{a+b}{2}\right], \\
\int_{b}^{t}\left[(h(b)-h(s))^{\alpha-1}-(h(s)-h(a))^{\alpha-1}\right. & \\
& \left.+(h(b)-h(a))^{\alpha-1}\right] g(s) h^{\prime}(s) \mathrm{d} s \quad t \in\left[\frac{a+b}{2}, b\right] .
\end{aligned}\right.
$$


Proof. It suffices to note that

$$
\begin{aligned}
I= & \int_{a}^{b} k(t) \mathrm{d} f(h(t)) \\
= & \int_{a}^{\frac{a+b}{2}}\left\{\int _ { a } ^ { t } \left[(h(b)-h(a))^{\alpha-1}-(h(b)-h(s))^{\alpha-1}\right.\right. \\
& \left.\left.\quad+(h(s)-h(a))^{\alpha-1}\right] g(s) h^{\prime}(s) \mathrm{d} s\right\} \mathrm{d} f(h(t)) \\
+ & \int_{\frac{a+b}{2}\left\{\int _ { b } ^ { b } \left[(h(b)-h(s))^{\alpha-1}-(h(s)-h(a))^{\alpha-1}\right.\right.}^{t} \\
= & \left.\left.\quad+(h(b)-h(a))^{\alpha-1}\right] g(s) h^{\prime}(s) \mathrm{d} s\right\} \mathrm{d} f(h(t)) \\
& I_{1}+I_{2} .
\end{aligned}
$$

By integration by parts, we get

$$
\begin{gathered}
I_{1}=\int_{a}^{\frac{a+b}{2}}\left\{\int _ { a } ^ { t } \left[(h(b)-h(a))^{\alpha-1}-(h(b)-h(s))^{\alpha-1}\right.\right. \\
\left.\left.\quad+(h(s)-h(a))^{\alpha-1}\right] g(s) h^{\prime}(s) \mathrm{d} s\right\} \mathrm{d} f(h(t)) \\
=\left\{\int _ { a } ^ { t } \left[(h(b)-h(a))^{\alpha-1}-(h(b)-h(s))^{\alpha-1}\right.\right. \\
\left.\left.+(h(s)-h(a))^{\alpha-1}\right] g(s) h^{\prime}(s) \mathrm{d} s\right\}\left.f(h(t))\right|^{\frac{a+b}{2}} \\
\quad-\left\{\int _ { a } ^ { \frac { a + b } { 2 } } \left[(h(b)-h(a))^{\alpha-1}-(h(b)-h(t))^{\alpha-1}\right.\right. \\
\left.\left.+(h(t)-h(a))^{\alpha-1}\right] g(t) f(h(t)) h^{\prime}(t) \mathrm{d} t\right\} \\
=f\left(h\left(\frac{a+b}{2}\right)\right)\left\{\int _ { a } ^ { \frac { a + b } { 2 } } \left[(h(b)-h(a))^{\alpha-1}-(h(b)-h(s))^{\alpha-1} .\right.\right. \\
\left.\left.(h(s)-h(a))^{\alpha-1}\right] g(s) h^{\prime}(s) \mathrm{d} s\right\}
\end{gathered}
$$




$$
\begin{gathered}
-\left\{\int _ { a } ^ { \frac { a + b } { 2 } } \left[(h(b)-h(a))^{\alpha-1}-(h(b)-h(t))^{\alpha-1}\right.\right. \\
\left.\left.+(h(t)-h(a))^{\alpha-1}\right] g(t) f(h(t)) h^{\prime}(t) \mathrm{d} t\right\} \\
=\Gamma(\alpha)\left[f ( h ( \frac { a + b } { 2 } ) ) \left\{J_{\left(\frac{a+b}{2}\right)^{-}}^{\alpha}(h(a))-J_{a^{+}}^{\alpha} g(h(b))\right.\right. \\
\left.+\frac{1}{\Gamma(\alpha)} \int_{a}^{\frac{a+b}{2}}(h(b)-h(a))^{\alpha-1} g(s) h^{\prime}(s) \mathrm{d} s\right\} \\
+\quad J_{a^{+}}^{\alpha}(g(f \circ h))(b)-J_{\left(\frac{a+b}{2}\right)^{-}}^{\alpha}(g(f \circ h))(a) \\
\left.-\frac{1}{\Gamma(\alpha)} \int_{a}^{\frac{a+b}{2}}(h(b)-h(a))^{\alpha-1} g(t) f(h(t)) h^{\prime}(t) \mathrm{d} t\right]
\end{gathered}
$$

and similarly

$$
\begin{aligned}
& I_{2}=\int_{\frac{a+b}{2}}^{b}\left\{\int _ { b } ^ { t } \left[(h(b)-h(s))^{\alpha-1}-(h(s)-h(a))^{\alpha-1}\right.\right. \\
& \left.\left.+(h(b)-h(a))^{\alpha-1}\right] g(s) h^{\prime}(s) \mathrm{d} s\right\} \mathrm{d} f(h(t)) \\
& =\left\{\int _ { b } ^ { t } \left[(h(b)-h(s))^{\alpha-1}-(h(s)-h(a))^{\alpha-1}\right.\right. \\
& \left.\left.+(h(b)-h(a))^{\alpha-1}\right] g(s) h^{\prime}(s) \mathrm{d} s\right\}\left.f(h(t))\right|_{\frac{a+b}{2}} ^{b} \\
& -\left\{\int _ { \frac { a + b } { 2 } } ^ { b } \left[(h(b)-h(t))^{\alpha-1}-(h(t)-h(a))^{\alpha-1}\right.\right. \\
& \left.\left.+(h(b)-h(a))^{\alpha-1}\right] g(t) f(h(t)) h^{\prime}(t) \mathrm{d} t\right\} \\
& =f\left(h\left(\frac{a+b}{2}\right)\right)\left\{\int _ { \frac { a + b } { 2 } } ^ { b } \left[(h(b)-h(s))^{\alpha-1}-(h(s)-h(a))^{\alpha-1}\right.\right. \\
& \left.\left.+(h(b)-h(a))^{\alpha-1}\right] g(s) h^{\prime}(s) \mathrm{d} s\right\}
\end{aligned}
$$




$$
\begin{aligned}
-\left\{\int_{\frac{a+b}{2}}^{b}[\right. & (h(b)-h(t))^{\alpha-1}-(h(t)-h(a))^{\alpha-1} \\
& \left.\left.+(h(b)-h(a))^{\alpha-1}\right] g(t) f(h(t)) h^{\prime}(t) \mathrm{d} t\right\} \\
=\Gamma(\alpha)\left[f ( h ( \frac { a + b } { 2 } ) ) \left\{J_{\left(\frac{a+b}{2}\right)^{+}}^{\alpha} g(h(b))-J_{b^{-}}^{\alpha} g(h(a))\right.\right. & \\
& \left.+\frac{1}{\Gamma(\alpha)} \int_{\frac{a+b}{2}}^{b}(h(b)-h(a))^{\alpha-1} g(s) h^{\prime}(s) \mathrm{d} s\right\} \\
& -\left\{J_{\left(\frac{a+b}{2}\right)^{+}}^{+}(g(f \circ h))(b)+J_{b^{-}}^{\alpha}(g(f \circ h))(a)\right. \\
& \left.-\frac{1}{\Gamma(\alpha)} \int_{\frac{a+b}{2}}^{b}(h(b)-h(a))^{\alpha-1} g(t) f(h(t)) h^{\prime}(t) \mathrm{d} t\right\} .
\end{aligned}
$$

Thus, can write

$$
\begin{aligned}
& I_{1}+I_{2}=\Gamma(\alpha)\left\{f ( h ( \frac { a + b } { 2 } ) ) \left[-J_{a^{+}}^{\alpha} g(h(b))-J_{b^{-}}^{\alpha} g(h(a))\right.\right. \\
& +J_{\left(\frac{a+b}{2}\right)^{\alpha}}^{+} g(h(b))+J_{\left(\frac{a+b}{2}\right)^{\alpha}}^{\alpha} g(h(a)) \\
& +\frac{1}{\Gamma(\alpha)} \int_{a}^{\frac{a+b}{2}}(h(b)-h(a))^{\alpha-1} g(s) h^{\prime}(s) \mathrm{d} s \\
& \left.+\frac{1}{\Gamma(\alpha)} \int_{\frac{a+b}{2}}^{b}(h(b)-h(a))^{\alpha-1} g(s) h^{\prime}(s) \mathrm{d} s\right] \\
& +J_{b^{-}}^{\alpha}(g(f \circ h))(a)+J_{a^{+}}^{\alpha}(g(f \circ h))(b) \\
& -J_{\left(\frac{a+b}{2}\right)^{-}}^{\alpha}(g(f \circ h))(a)-J_{\left(\frac{a+b}{2}\right)^{+}}^{\alpha}(g(f \circ h))(b) \\
& -\frac{1}{\Gamma(\alpha)} \int_{a}^{\frac{a+b}{2}}(h(b)-h(a))^{\alpha-1} g(t) f(h(t)) h^{\prime}(t) \mathrm{d} t \\
& \left.-\frac{1}{\Gamma(\alpha)} \int_{\frac{a+b}{2}}^{b}(h(b)-h(a))^{\alpha-1} g(t) f(h(t)) h^{\prime}(t) \mathrm{d} t\right\}
\end{aligned}
$$


Multiplying the both sides $(\Gamma(\alpha))^{-1}$, we obtain (14) which completes the proof.

Remark 2.6. If we choose $h(x)=x$ in Lemma 2.2, we have

$$
\begin{aligned}
& \left\{f ( \frac { a + b } { 2 } ) \left[-J_{a^{+}}^{\alpha} g(b)-J_{b^{-}}^{\alpha} g(a)+J_{\left(\frac{a+b}{2}\right)^{+}}^{\alpha} g(b)+J_{\left(\frac{a+b}{2}\right)^{-}}^{\alpha} g(a)\right.\right. \\
& \left.\quad+\frac{(b-a)^{\alpha-1}}{\Gamma(\alpha)} \int_{a}^{b} g(s) \mathrm{d} s\right]+J_{a^{+}}^{\alpha}(g f)(b)+J_{b^{-}}^{\alpha}(g f)(a) \\
& \left.\quad-J_{\left(\frac{a+b}{2}\right)^{+}}^{\alpha}(g f)(b)-J_{\left(\frac{a+b}{2}\right)^{-}}^{\alpha}(g f)(a)-\frac{(b-a)^{\alpha-1}}{\Gamma(\alpha)} \int_{a}^{b} g(t) f(t) \mathrm{d} t\right\} \\
& =\frac{1}{\Gamma(\alpha)} \int_{a}^{b} k(t) \mathrm{d} f(t)
\end{aligned}
$$

where

$$
k(t)=\left\{\begin{array}{l}
\left.\int_{a}^{t}[(b-a))^{\alpha-1}-(b-s)^{\alpha-1}+(s-a)^{\alpha-1}\right] g(s) \mathrm{d} s, \quad t \in\left[a, \frac{a+b}{2}\right], \\
\int_{b}^{t}\left[(b-s)^{\alpha-1}-(s-a)^{\alpha-1}+(b-a)^{\alpha-1}\right] g(s) \mathrm{d} s, \quad t \in\left[\frac{a+b}{2}, b\right] .
\end{array}\right.
$$

Remark 2.7. If we choose $h(x)=x$ and $g(x)=1$ in Lemma 2.2, we have

$$
\begin{aligned}
& \left\{f\left(\frac{a+b}{2}\right)\left[(b-a)^{\alpha}\left(1+\frac{1}{\alpha}\left(\frac{1}{2^{\alpha}}-2\right)\right)\right]+J_{a^{+}}^{\alpha} f(b)+J_{b^{-}}^{\alpha} f(a)\right. \\
& \left.\quad-J_{\left(\frac{a+b}{2}\right)^{+}}^{\alpha} f(b)-J_{\left(\frac{a+b}{2}\right)^{-}}^{\alpha} f(a)-\frac{(b-a)^{\alpha-1}}{\Gamma(\alpha)} \int_{a}^{b} f(t) \mathrm{d} t\right\} \\
& =\frac{1}{\Gamma(\alpha)} \int_{a}^{b} k(t) \mathrm{d} f(t)
\end{aligned}
$$

where

$$
k(t)= \begin{cases}\int_{a}^{t}\left[(b-a)^{\alpha-1}-(b-s)^{\alpha-1}+(s-a)^{\alpha-1}\right] \mathrm{d} s, & t \in\left[a, \frac{a+b}{2}\right], \\ \int_{b}^{t}\left[(b-s)^{\alpha-1}-(s-a)^{\alpha-1}+(b-a)^{\alpha-1}\right] \mathrm{d} s, & t \in\left[\frac{a+b}{2}, b\right] .\end{cases}
$$


Remark 2.8. If we choose $h(x)=x, \alpha=1$ and $g(x)=1$ in Lemma 2.2, we obtain Lemma 2.1 in [21]

$$
\begin{aligned}
& \frac{1}{b-a} \int_{a}^{b} f(t) \mathrm{d} t-f\left(\frac{a+b}{2}\right) \\
= & (b-a)\left(\int_{0}^{\frac{1}{2}} t f^{\prime}(t a+(1-t) b) \mathrm{d} t+\int_{\frac{1}{2}}^{1}(t-1) f^{\prime}(t a+(1-t) b) \mathrm{d} t\right) .
\end{aligned}
$$

Theorem 2.3. Let $f: I \rightarrow \mathbb{R}$ be a differentiable mapping on $I^{\circ}$ and $f^{\prime} \in$ $X_{h}^{p}[a, b]$ with $a<b$ and $g:[a, b] \rightarrow \mathbb{R}$ is continuous. If $\left|f^{\prime}\right|$ is convex on $[a, b]$, then the following inequality for fractional integrals holds:

$$
\begin{aligned}
& \left\{f ( \frac { a + b } { 2 } ) \left[-J_{a^{+}}^{\alpha} g(b)-J_{b^{-}}^{\alpha} g(a)+J_{\left(\frac{a+b}{2}\right)^{+}}^{\alpha} g(b)\right.\right. \\
& \left.+J_{\left(\frac{a+b}{2}\right)^{-}}^{\alpha} g(a)+\frac{(b-a)^{\alpha-1}}{\Gamma(\alpha)} \int_{a}^{b} g(s) \mathrm{d} s\right] \\
& +J_{a^{+}}^{\alpha}(g f)(b)+J_{b^{-}}^{\alpha}(g f)(a)-J_{\left(\frac{a+b}{2}\right)^{+}}^{\alpha}(g f)(b) \\
& \left.-J_{\left(\frac{a+b}{2}\right)^{-}}^{\alpha}(g f)(a)-\frac{(b-a)^{\alpha-1}}{\Gamma(\alpha)} \int_{a}^{b} g(t) f(t) \mathrm{d} t\right\} \\
& \leq \frac{\|g\|_{\left[a, \frac{a+b}{2}\right], \infty}}{(b-a) \Gamma(\alpha)}\left\{\int_{a}^{\frac{a+b}{2}}\left[\int_{a}^{t}\left[(b-a)^{\alpha-1}-(b-s)^{\alpha-1}+(s-a)^{\alpha-1}\right] \mathrm{d} s\right]\right. \\
& +\frac{\|g\|_{\left[\frac{a+b}{2}, b\right], \infty}}{(b-a) \Gamma(\alpha)}\left\{\int_{\frac{a+b}{2}}^{b}\left[\int_{b}^{t}\left[(b-s)^{\alpha-1}-(s-a)^{\alpha-1}+(b-a)^{\alpha-1}\right] g(s) \mathrm{d} s\right]\right. \\
& \left.\times\left((b-t)\left|f^{\prime}(a)\right|+(t-a)\left|f^{\prime}(b)\right|\right) \mathrm{d} t\right\}
\end{aligned}
$$

with $\alpha>0$.

Proof. If $\left|f^{\prime}\right|$ is convex on $[a, b]$, we know that for $t \in[a, b]$

$$
\left|f^{\prime}(t)\right|=\left|f^{\prime}\left(\frac{b-t}{b-a} a+\frac{t-a}{b-a} b\right)\right| \leq \frac{b-t}{b-a}\left|f^{\prime}(a)\right|+\frac{t-a}{b-a}\left|f^{\prime}(b)\right| .
$$


From Remark 2.6, we have

$$
\begin{aligned}
& \left\{f ( \frac { a + b } { 2 } ) \left[-J_{a^{+}}^{\alpha} g(b)-J_{b^{-}}^{\alpha} g(a)+J_{\left(\frac{a+b}{2}\right)^{+}}^{\alpha} g(b)+J_{\left(\frac{a+b}{2}\right)^{-}}^{\alpha} g(a)\right.\right. \\
& \left.+\frac{(b-a)^{\alpha-1}}{\Gamma(\alpha)} \int_{a}^{b} g(s) \mathrm{d} s\right]+J_{a^{+}}^{\alpha}(g f)(b)+J_{b^{-}}^{\alpha}(g f)(a) \\
& \left.-J_{\left(\frac{a+b}{2}\right)^{+}}^{\alpha}(g f)(b)-J_{\left(\frac{a+b}{2}\right)^{-}}^{\alpha}(g f)(a)-\frac{(b-a)^{\alpha-1}}{\Gamma(\alpha)} \int_{a}^{b} g(t) f(t) \mathrm{d} t\right\} \\
& \leq \frac{1}{\Gamma(\alpha)}\left\{\int_{a}^{\frac{a+b}{2}}\left|\int_{a}^{t}\left[(b-a)^{\alpha-1}-(b-s)^{\alpha-1}+(s-a)^{\alpha-1}\right] g(s) \mathrm{d} s\right|\left|f^{\prime}(t)\right| \mathrm{d} t\right. \\
& \left.+\int_{\frac{a+b}{2}}^{b}\left|\int_{b}^{t}\left[(b-s)^{\alpha-1}-(s-a)^{\alpha-1}+(b-a)^{\alpha-1}\right] \mathrm{d} s\right| g(s)\left|f^{\prime}(t)\right| \mathrm{d} t\right\} \\
& \leq \frac{1}{(b-a) \Gamma(\alpha)}\left\{\int_{a}^{\frac{a+b}{2}}\left[\int_{a}^{t}\left[(b-a)^{\alpha-1}-(b-s)^{\alpha-1}+(s-a)^{\alpha-1}\right] g(s) \mathrm{d} s\right]\right. \\
& \times\left((b-t)\left|f^{\prime}(a)\right|+(t-a)\left|f^{\prime}(b)\right|\right) \mathrm{d} t \\
& +\int_{\frac{a+b}{2}}^{b}\left[\int_{b}^{t}\left[(b-s)^{\alpha-1}-(s-a)^{\alpha-1}+(b-a)^{\alpha-1}\right] g(s) \mathrm{d} s\right] \\
& \left.\times\left((b-t)\left|f^{\prime}(a)\right|+(t-a)\left|f^{\prime}(b)\right|\right) \mathrm{d} t\right\} \\
& \leq \frac{\|g\|_{\left[a, \frac{a+b}{2}\right], \infty}}{(b-a) \Gamma(\alpha)}\left\{\int_{a}^{\frac{a+b}{2}}\left[\int_{a}^{t}\left[(b-a)^{\alpha-1}-(b-s)^{\alpha-1}+(s-a)^{\alpha-1}\right] \mathrm{d} s\right]\right. \\
& \left.\times\left((b-t)\left|f^{\prime}(a)\right|+(t-a)\left|f^{\prime}(b)\right|\right) \mathrm{d} t\right\} \\
& +\frac{\|g\|_{\left[\frac{a+b}{2}, b\right], \infty}}{(b-a) \Gamma(\alpha)}\left\{\int_{\frac{a+b}{2}}^{b}\left[\int_{b}^{t}\left[(b-s)^{\alpha-1}-(s-a)^{\alpha-1}+(b-a)^{\alpha-1}\right] g(s) \mathrm{d} s\right]\right. \\
& \left.\times\left((b-t)\left|f^{\prime}(a)\right|+(t-a)\left|f^{\prime}(b)\right|\right) \mathrm{d} t\right\}
\end{aligned}
$$




$$
\begin{gathered}
\leq \frac{\|g\|_{\left[a, \frac{a+b}{2}\right], \infty}\left\{\int_{a}^{\frac{a+b}{2}}\left[\int_{a}^{t}\left[(b-a)^{\alpha-1}-(b-s)^{\alpha-1}+(s-a)^{\alpha-1}\right] \mathrm{d} s\right]\right.}{(b-a) \Gamma} \\
\left.\times\left((b-t)\left|f^{\prime}(a)\right|+(t-a)\left|f^{\prime}(b)\right|\right) \mathrm{d} t\right\} \\
+\frac{\|g\|_{\left[\frac{a+b}{2}, b\right], \infty}}{(b-a) \Gamma(\alpha)}\left\{\int_{\frac{a+b}{2}}^{b}\left[\int_{b}^{t}\left[(b-s)^{\alpha-1}-(s-a)^{\alpha-1}+(b-a)^{\alpha-1}\right] g(s) \mathrm{d} s\right]\right. \\
\left.\times\left((b-t)\left|f^{\prime}(a)\right|+(t-a)\left|f^{\prime}(b)\right|\right) \mathrm{d} t\right\} .
\end{gathered}
$$

This completes the proof.

\section{REFERENCES}

[1] A.A. Kilbas, H.M. Srivastava, J.J. Trujillo, Theory and Applications of Fractional Differantial Equations, Elsevier B.V., Amsterdam, 2006.

[2] A. Akkurt, H. Ylldırım, On Feng Qi Type Integral Inequalities For Generalized Fractional Integrals, IAAOJ, Scientific Science, 1(2), (2013), 22-25.

[3] A. Akkurt, Z. Kırtay, H. Yıldırım, Generalized Fractional Integrals Inequalities for Continuous Random Variables, Journal of Probability and Statistics, Volume 2015 (2015), Article ID 958980, 7 pages, http://dx.doi.org/10.1155/2015/958980.

[4] E. F. Beckenbach, Convex functions, Bull. Amer. Math. Soc., 54 (1948) 439-460. http://dx.doi.org/10.1090/s0002-9904-1948-08994-7

[5] E. Set, İ. İşcan, M. E. Özdemir and M. Z. Sarıkaya, On new Hermite-Hadamard-Fejer type inequalities for convex functions via fractional integrals, Applied Mathematics and Computation, 259(2015), 875-881.

[6] H. Yıldırım, Z. Kırtay, Ostrowski Inequality for Generalized Fractional Integral and Related Inequalities, Malaya Journal of Matematik: Volume 2, Issue 3, 2014, pp. 322-329.

[7] İ. İşcan, Hermite-Hadamard-Fejér type inequalities for convex functions via fractional integrals, Stud. Univ. Babeş-Bolyai Math. 60 (2015), No. 3, 355-366

[8] J. Park, Inequalities of Hermite-Hadamard-Fejér Type for Convex Functions via Fractional Integrals,International Journal of Mathematical Analysis,Vol. 8, 2014, no. 59, pp. 2927-2937, http://dx.doi.org/10.12988/ijma.2014.411378.

[9] K. L. Tseng, S. R. Hwang, S. S. Dragomir, Fejér-type inequalities (I), J. Inequal. Appl., 2010 (2010), Art ID: 531976, 7 pages. http://dx.doi.org/10.1155/2010/531976.

[10] L. Fejér, Uberdie Fourierreihen, II, Math. Naturwise. Anz Ungar. Akad., Wiss 24 (1906) 369-390 (in Hungarian). 
[11] M.Z. Sarikaya, E. Set, H. Yaldız, N. Başak, Hermite-Hadamard's inequalities for fractional integrals and related fractional inequalities, Math. Comput.Modell. 57 (9) (2013) 2403-2407.

[12] M. E. Özdemir, M. Avic, H. Kavurmaci, Hermite-Hadamard type inequalities for sconvex and s-concave functions via fractional integrals, Turkish Journal of Science 1 (2016): 28-40.

[13] M. Z. Sarikaya, On new Hermite Hadamard Fejér type integral inequalities, Stud. Univ. Babes-Bolyai Math. 57(3) (2012) 377-386.

[14] M. Tunç, On some new inequalities for convex functions, Turk. J. Math., 35 (2011), $1-7$.

[15] M. Z. Sarikaya, S. Erden, On the Hermite-Hadamard-Fejér type integral inequality for convex function, Turk. J. Anal. Number Theory 2(3) (2014) 85-89.

[16] M. Z. Sarikaya, A. Sağlam and H. Yıldırım, On some Hadamard-type inequalities for h-convex functions, J. Math. Ineq., 2(3) (2008), 335-341.

[17] S.G. Samko, A.A. Kilbas, and O.I. Marichev, Fractional Integrals and Derivatives Theory and Applications, Gordon and Breach, Linghorne, 1993.

[18] S. Belarbi, Z. Dahmani, On some new fractional integral inequalities, J. Ineq. Pure Appl. Math. 10 (3) (2009). Art. 86.

[19] S. Kılınç, H. Yıldırım, Generalized Fractional Integral Inequalities Involving Hipergeometric Operators, IJPAM., vol.101 no.1 (2015) pp.71-82.

[20] Z. Dahmani, On Minkowski and Hermite-Hadamard integral inequalities via fractional integration, Ann. Funct. Anal. 1(1) (2010) 51-58.

[21] U.S. Kırmac1, Inequalities for differentiable mappings and applications to special means of real numbers and to midpoint formula, Appl. Math. Comput. 147 (1) (2004) 137-146.

\author{
Abdullah Akmurt \\ Department of Mathematics \\ University of Kahramanmaraş SÜtÇÜ İmam \\ KaHRAMANMARAŞ 46100 \\ TURKEY \\ E-mail address: abdullahmat@gmail.com \\ HüSEYIN YILDIRIM \\ Department of Mathematics \\ UnIVERSITY of Kahramanmaraş SÜtÇÜ İmaM \\ KAHRAMANMARAŞ 46100 \\ TURKEY \\ E-mail address: hyildir@ksu.edu.tr
}

